Check for updates

Cite this: RSC Adv., 2019, 9, 37686

Received 26th July 2019

Accepted 12th November 2019

DOI: 10.1039/c9ra05793e

rsc.li/rsc-advances

\section{Removal of methylene blue dye by solvothermally reduced graphene oxide: a metal-free adsorption and photodegradation method $\dagger$}

\author{
Valerie Ling Er Siong, (D) ${ }^{a}$ Kian Mun Lee, ${ }^{a}$ Joon Ching Juan, (D) ${ }^{a}$ Chin Wei Lai, (D) *a \\ Xin Hong Tai (D) and Cheng Seong Khe (D) ${ }^{\mathrm{b}}$
}

In this work, reduced graphene oxide ( $\mathrm{rGO}$ ) was fabricated at different reduction temperatures via an environmentally friendly solvothermal approach. The $\mathrm{rGO}$ formed at $160{ }^{\circ} \mathrm{C}$ clearly showed the partial restoration of the $\mathrm{sp}^{2}$ hybridization brought about by the elimination of oxygenated functionalities from the surface. Owing to the augmented surface area and the band gap reduction, rGO-160 exhibited the best adsorption (29.26\%) and photocatalytic activity (32.68\%) towards the removal of MB dye. The effects of catalyst loading, initial concentration of dye, light intensity, and initial pH of solution were evaluated. It was demonstrated that rGO-160 could achieve a higher adsorptive removal (87.39\%) and photocatalytic degradation (98.57\%) of MB dye when $60 \mathrm{mg}$ of catalyst, $50 \mathrm{ppm}$ of dye at $\mathrm{pH} 11$, and $60 \mathrm{~W} \mathrm{~m}^{-2}$ of UV$\mathrm{C}$ light source were used. The MB photodegradation activity of $\mathrm{rGO}-160$ displayed no obvious decrease after five successive cycles. This study provides a potential metal-free adsorbent-cum-photocatalyst for the decontamination of dyes from wastewater.

\section{Introduction}

Dyes play an important part in different areas of the textile manufacturing industries. Nevertheless, the dyes are nonbiodegradable, and are often released into the water environment without proper precautions. ${ }^{1}$ Most of the dyes possess toxic and carcinogenic properties, which can have deleterious effects on human health and ecosystems. ${ }^{2}$ Dyes are stable towards natural degradation processes due to their intricate aromatic structures. ${ }^{3}$ For this reason, the removal of dyes has been accomplished by various treatment techniques. The adsorption process is by far the most common approach used in industry, due to the accessibility of inexpensive adsorbents with high adsorption capacities. ${ }^{4}$ The photocatalysis process is yet another popular method used due to its easiness of application. ${ }^{5}$ Generally, both superoxide $\left(\cdot \mathrm{O}_{2}{ }^{-}\right)$and hydroxyl $(\cdot \mathrm{OH})$ radicals are widely accepted as the reactive oxygen species (ROS) in the photooxidation of dye pollutants. ${ }^{6}$ The elimination of dyes in both reactions is highly dependent on the surface area of the materials used. ${ }^{7}$

Being a type of chemically-altered graphene, reduced graphene oxide ( $\mathrm{rGO}$ ) is more economically fitted for extensive

${ }^{a}$ Nanotechnology \& Catalysis Research Centre, Institute for Advanced Studies, University of Malaya, Kuala Lumpur, Malaysia. E-mail: cwlai@um.edu.my

${ }^{b}$ Department of Fundamental and Applied Sciences, Universiti Teknologi PETRONAS, Perak Darul Ridzuan, Malaysia

$\dagger$ Electronic supplementary information (ESI) available. See DOI: $10.1039 /$ c9ra05793e production than pristine graphene. ${ }^{8}$ Commonly, rGO finds its application in the manufacture of graphene-based composites. ${ }^{9}$ rGO can be fabricated in various ways, including microwave, thermal, photo-thermal, chemical, photo-chemical, as well as microbial/bacterial processes. ${ }^{10}$ Chemical method appears to be advantageous owing to its affordability, simplicity, and extensive production. ${ }^{11}$ Particularly, there are three main steps in preparing rGO via this method. The initial step refers to the formation of graphite oxide from graphite by oxidation, whereby the surficial oxygen functionalities are introduced to the graphene layers. Due to the presence of oxygenated functionalities like carboxyls, hydroxyls and epoxides on the surface, graphite oxide is able to disperse in polar solvents, thereby forming stable dispersions. Subsequently, graphite oxide is exfoliated by either sonication or mechanical stirring to create graphene oxide (GO) with single or few layers. Lastly, GO is reduced to rGO by eliminating the surficial oxygen functionalities. ${ }^{12}$

Carbon-based materials have been traditionally utilized in the adsorption reactions to eliminate the organic and inorganic contaminants. ${ }^{13,14}$ One of the most popular adsorbents is the activated carbon. ${ }^{15}$ Recently, rGO has shown increasing usage in dye adsorption application. ${ }^{16,17}$ Owing to the presence of remaining surficial oxygen functionalities, along with some defects in the graphitic domains, rGO is deemed efficient for the adsorptive removal of dyes. ${ }^{18}$ Generally, rGO interacts with dyes through $\pi-\pi$ interaction, electrostatic interaction, hydrophobic association and structural conjugation. These interactions enable a wide range of dyes to adsorb on $\mathrm{rGO}^{19}$ In order to 
improve the dye adsorption capacity, rGO with large surface area, together with high porosity, is preferable, and this is achievable by controlling the quality of the GO precursor and the reduction method used. ${ }^{20}$

Today, the photocatalytic degradation of dyes has been used as a tool for manifesting the technological benefits of photocatalysis. Generally, when the band gap of a photocatalyst is equal to or lower than the energy of light absorbed, electrons are excited, thereby forming electrons and holes. The surrounding oxygen and water molecules are then utilized by the photogenerated electron-hole pairs to produce ROS, which are responsible to break down the dye molecules. ${ }^{21}$ There have been various studies reporting the usage of rGO in the elimination of dye pollutants by means of photocatalysis, especially the removal of harmful methylene blue (MB) dye from wastewater (Table S1 $\dagger$ ). In spite of the fact that rGO exhibited outstanding MB adsorption ability, there is a lack of information about the optimization of its photoactivity. Since rGO behaves like a semiconductor, plus the fact that it is inexpensive and friendly to the environment, there is a great potential for utilizing rGO as a metal-free photocatalyst for environmental remediation.

In this work, $\mathrm{rGO}$ with excellent adsorption properties and photoactivity towards the removal of MB dye was synthesized via an eco-friendly and simple solvothermal technique without employing any toxic reducing agents. The optimization of extraneous factors such as amount of catalyst, initial dye concentration, intensity of light, and $\mathrm{pH}$ were investigated.

\section{Experiment}

\subsection{Materials}

Synthetic graphite powder with the size of $<20 \mu \mathrm{m}$ was acquired from Sigma Aldrich. Methylene blue (MB, C.I. 52015, $\geq 99 \%$ ), hydrochloric acid ( $\mathrm{HCl}, \geq 37 \%)$, sulphuric acid $\left(\mathrm{H}_{2} \mathrm{SO}_{4}, \geq 95-\right.$ $97 \%)$, hydrogen peroxide $\left(\mathrm{H}_{2} \mathrm{O}_{2}, \geq 30 \%\right)$, and potassium permanganate $\left(\mathrm{KMnO}_{4}, \geq 99 \%\right)$ were obtained from Friendemann Schmidt. Di-phosphorus pentoxide $\left(\mathrm{P}_{2} \mathrm{O}_{5}, \geq 99 \%\right)$, potassium peroxodisulphate $\left(\mathrm{K}_{2} \mathrm{~S}_{2} \mathrm{O}_{8}, \geq 99 \%\right)$, and ethanol $\left(\mathrm{C}_{2} \mathrm{H}_{5} \mathrm{OH}, \geq 99.9 \%\right)$ were acquired from Merck. The materials used were all of analytical grade. In all experiments, deionized water $(18.2 \mathrm{M} \Omega \mathrm{cm})$ was employed.

\subsection{Pre-treatment of graphite powder}

Initially, graphite $(3 \mathrm{~g})$ was added into $\mathrm{H}_{2} \mathrm{SO}_{4}(12 \mathrm{ml})$ containing $\mathrm{P}_{2} \mathrm{O}_{5}(2.5 \mathrm{~g})$ and $\mathrm{K}_{2} \mathrm{~S}_{2} \mathrm{O}_{8}(2.5 \mathrm{~g}) .^{22}$ The mixture was stirred for a period of 4.5 hours at $80^{\circ} \mathrm{C}$ in an oil bath. Then, the mixture was gradually cooled down to ambient temperature. The mixture was kept aside for one night with the addition of deionized water $(500 \mathrm{ml})$. After vacuum filtration, the residue was washed with deionized water prior to drying.

\subsection{Preparation of graphene oxide (GO)}

A modified Hummers' method was used to oxidize graphite to $\mathrm{GO}^{23}$ In a typical process, pre-treated graphite was mixed with $\mathrm{H}_{2} \mathrm{SO}_{4}(120 \mathrm{ml})$ in an ice bath and vigorously stirred. The mixture was gradually added with $\mathrm{KMnO}_{4}(15 \mathrm{~g})$ and kept at
$35{ }^{\circ} \mathrm{C}$ for 2 hours. The stirring was continued for another 2 hours after deionized water $(250 \mathrm{ml})$ was added to the mixture. Then, deionized water $(500 \mathrm{ml})$ was added again, before adding $30 \% \mathrm{H}_{2} \mathrm{O}_{2}(20 \mathrm{ml})$ solution to cease the reaction. After the mixture was centrifuged, the GO precipitate obtained was cleansed with $10 \%(\mathrm{v} / \mathrm{v}) \mathrm{HCl}$ solution, followed by deionized water via centrifugation until a final $\mathrm{pH}$ of about 4 was obtained. Finally, GO was dried, pestled, and stored.

\subsection{Preparation of reduced graphene oxide (rGO)}

An eco-friendly and facile solvothermal approach was used to reduce $\mathrm{GO}$ to $\mathrm{rGO}$. Initially, $\mathrm{GO}(200 \mathrm{mg})$ was mixed with deionized water $(10 \mathrm{ml})$ and $\mathrm{C}_{2} \mathrm{H}_{5} \mathrm{OH}(20 \mathrm{ml})$. A stable GO dispersion was obtained after 30 minute of rigorous sonication of the mixture. Then, the mixture was transferred into an $80 \mathrm{ml}$ Teflon-lined stainless-steel autoclave, and heated at different temperatures $\left(80,120,160\right.$ and $\left.180^{\circ} \mathrm{C}\right)$ for 2 hours. The samples were labelled accordingly based on the denotation of rGO- $x$, where $x$ represents the studied reduction temperatures. Subsequently, the as-obtained rGO samples were filtered, rinsed, and dried.

\subsection{Methods of characterization}

The ordered and disordered crystalline structures of GO and rGOs were determined via Raman spectroscopy by employing Renishaw inVia Raman Microscope (Gloucestershire, UK) equipped with Ar-ion laser $(\lambda=514 \mathrm{~nm})$ over a scan range of $100-3200 \mathrm{~cm}^{-1}$. The elemental atomic composition of GO and rGOs were analyzed via energy dispersive X-ray spectroscopy (EDX) by using FEI Quanta FEG 650 EDX Unit (Oregon, USA). The crystalline phases of GO and rGOs were investigated via $\mathrm{X}$ ray diffraction (XRD) by using PANalytical X-ray Diffractometer (Almelo, Netherlands) with $\mathrm{Cu} \mathrm{K} \alpha(\lambda=0.15406 \mathrm{~nm})$ radiation over a scan rate of $0.02^{\circ} \mathrm{s}^{-1}$ at a $2 \theta$ range of $10-70^{\circ}$ under $40 \mathrm{kV} /$ $30 \mathrm{~mA}$. Brunauer-Emmett-Teller (BET) and Barrett-JoynerHalenda (BJH) analyses were carried out in $\mathrm{N}_{2}$ atmosphere at 77 K using Micromeritics ASAP 2020 Surface Area and Porosity Analyzer (Georgia, USA) to examine the specific surface areas, pore sizes, and pore volumes of GO and rGOs. Degassing of the samples was performed at $150{ }^{\circ} \mathrm{C}$ for 24 hours prior to measurement to remove moisture. Ultraviolet-visible (UV-vis) spectrophotometry was conducted by using PerkinElmer Lambda $35 \mathrm{UV} /$ vis Spectrophotometer (Massachusetts, USA) at an absorption range of $200-800 \mathrm{~nm}$ to elucidate the optical properties of GO and rGOs. The optical band gaps of GO and rGOs were then determined from the linear extrapolation of Tauc plots.

\subsection{Adsorption and photocatalytic activity measurements}

In a custom-made photoreactor (Fig. 1), GO/rGO (20 mg) suspended in $\mathrm{MB}$ solution $(50 \mathrm{ppm}, 100 \mathrm{ml})$ was aerated and stirred in darkness to determine the time needed to achieve dye adsorption-desorption equilibrium. Then, a new and similar suspension was allowed to reach dark adsorption and desorption equilibrium, followed by 6 hours of irradiation with UV-C light $\left(2 \times 95 \mathrm{~W}\right.$; intensity $\left.\approx 60 \mathrm{~W} \mathrm{~m}^{-2}\right)$. At certain intervals, 
aliquots were withdrawn, filtered, and subjected to UV-vis analysis at $664 \mathrm{~nm}$. The photocatalyst with the best adsorption and photoactivity was then optimized based on catalyst dosage, initial concentration of dye, light intensity, and $\mathrm{pH}$ of solution. In addition, control experiment was also conducted prior to adsorption and photocatalytic activity measurements, whereby MB solution (50 ppm, $100 \mathrm{ml}$ ) was irradiated with the same UV-C light in the absence of photocatalyst for 10 hours. Under optimized experimental conditions, the stability and reusability of the optimum rGO sample towards the MB photodegradation process were determined by repeating the reaction for up to five cycles.

The adsorption percentage $\left(\begin{array}{ll}\% & C_{\text {ads }}\end{array}\right)$ and the photodegradation efficiency ( $\% C_{\mathrm{deg}}$ ) were calculated accordingly by using the following equations:

$$
\begin{gathered}
\% C_{\text {ads }}=\frac{C_{\text {initial }}-C_{0}}{C_{\text {initial }}} \times 100 \% \\
\% C_{\mathrm{deg}}=\frac{C_{0}-C_{t}}{C_{0}} \times 100 \%, \quad 0 \leq t \leq 6
\end{gathered}
$$

where $C_{\text {initial }}, C_{0}$, and $C_{t}$ are the respective concentrations of MB dye at the initial, after reaching adsorption-desorption equilibrium, and at time, $t$. Subsequently, the photocatalytic degradation reaction was modeled by employing the pseudofirst-order kinetic equation as below:

$$
-\ln \left(\frac{C_{t}}{C_{0}}\right)=k t, \quad 0 \leq t \leq 6
$$

where $k\left(\mathrm{~h}^{-1}\right)$ denotes the rate constant for pseudo-first-order.

\section{Results and discussion}

\subsection{Characterization of GO and rGOs}

The Raman spectra of GO and rGOs are shown in Fig. 2. All samples exhibited both $\mathrm{D}\left(\sim 1353 \mathrm{~cm}^{-1}\right)$ and $\mathrm{G}\left(\sim 1591 \mathrm{~cm}^{-1}\right)$ bands of graphene. The $\mathrm{D}$ band referred to the breathing mode of $\mathrm{sp}^{2}$ carbon atoms in the hexagonal structure. ${ }^{24}$ It was resulted from the formation of structural defects. ${ }^{25}$ The $\mathrm{G}$ band reflected the vibration of $\mathrm{sp}^{2}$ carbon atoms in the aromatic rings. ${ }^{26}$ In contrast to D band, the G band demonstrated higher intensity, suggesting that the samples were not fully exfoliated. ${ }^{27}$

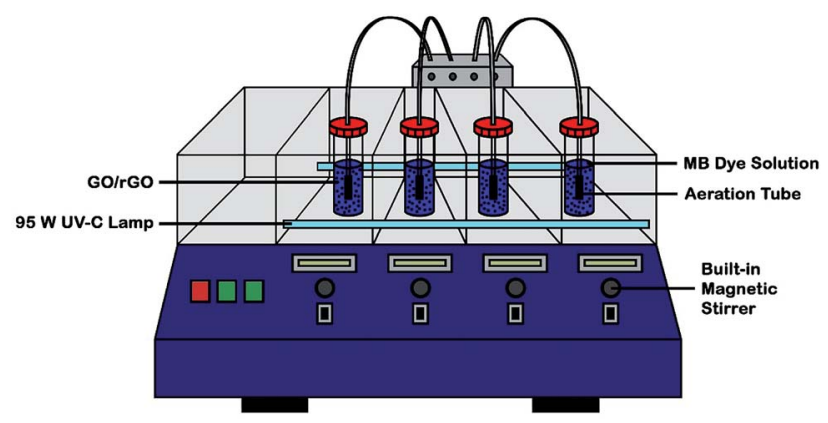

Fig. 1 Schematic diagram of custom-made UV-C photoreactor.

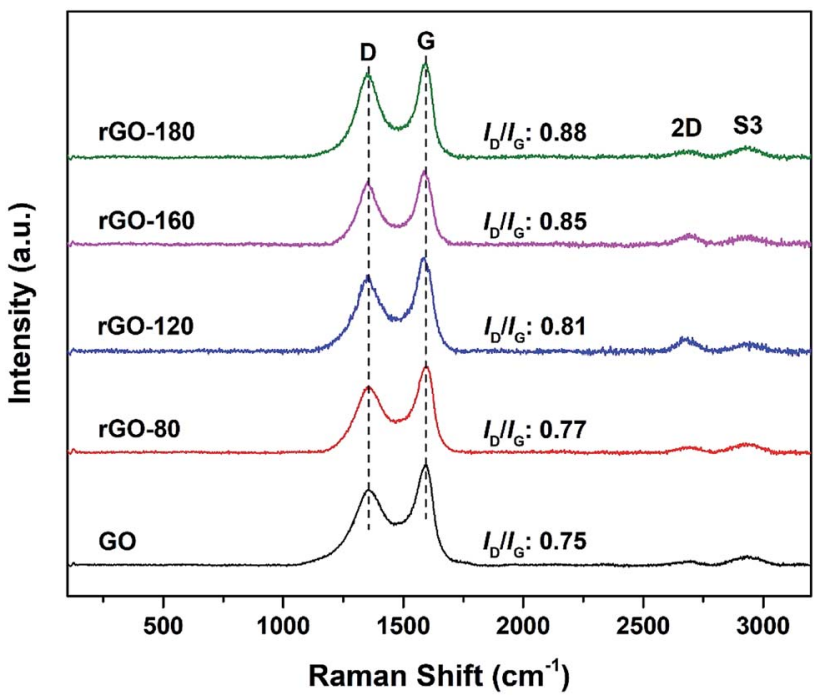

Fig. 2 Raman spectra of GO, rGO-80, rGO-120, rGO-160 and rGO180

The intensity ratio of the $\mathrm{D}$ to $\mathrm{G}$ band $\left(I_{\mathrm{D}} / I_{\mathrm{G}}\right.$ ratio $)$ can be used to gauge the degree of disorder and the change in average size of the $\mathrm{sp}^{2}$ fraction during the reduction of GO to rGO. ${ }^{28}$ In Fig. 2, the $I_{\mathrm{D}} / I_{\mathrm{G}}$ ratio increased steadily (from 0.75 of GO to 0.88 of rGO180) with higher reduction temperature, signifying an increased degree of reduction. ${ }^{\mathbf{2 9 , 3 0}}$ This can be due to more defects were created as the surficial oxygen functionalities were gradually eliminated during the solvothermal reduction process. ${ }^{31,32}$ The defects originated from surface ripples and edges, as a result of higher degree of defragmentation and smaller average $\mathrm{sp}^{2}$ domain size after reduction..$^{28,33}$ On the other hand, the $2 \mathrm{D}$ $\left(\sim 2684 \mathrm{~cm}^{-1}\right)$ and S3 $\left(\sim 2935 \mathrm{~cm}^{-1}\right)$ bands were also detected. The $2 \mathrm{D}$ band is attributed to the inelastic scattering from two phonons, whereas the $\mathrm{S} 3$ band is resulted from the combination of both $\mathrm{D}$ and $\mathrm{G}$ bands. $^{34}$ As the reduction temperature increased, the intensities of the two bands also slightly increased, indicating an enhanced degree of graphitization. ${ }^{35}$

Table 1 and Fig. S1† show the EDX elemental atomic analysis of GO and rGOs, where only carbon and oxygen were detected. The $\mathrm{C} / \mathrm{O}$ atomic ratio of GO is the lowest at 1.79 , which implied that higher oxygen content was present in GO. The value is comparable to the findings obtained in previous GO studies. ${ }^{36,37}$ After solvothermal reduction of $\mathrm{GO}$, the $\mathrm{C} / \mathrm{O}$ atomic ratio was

Table 1 EDX elemental analysis of GO, rGO-80, rGO-120, rGO-160 and rGO-180

\begin{tabular}{llll}
\hline & \multicolumn{2}{l}{ Elemental analysis (at\%) } \\
\cline { 2 - 4 } Sample & $\mathrm{C}$ & $\mathrm{O}$ & $\mathrm{C} / \mathrm{O}$ \\
\hline GO & 64.22 & 35.78 & 1.79 \\
rGO-80 & 65.02 & 34.98 & 1.86 \\
rGO-120 & 67.16 & 32.84 & 2.05 \\
rGO-160 & 80.24 & 19.76 & 4.06 \\
rGO-180 & 81.03 & 18.97 & 4.27
\end{tabular}




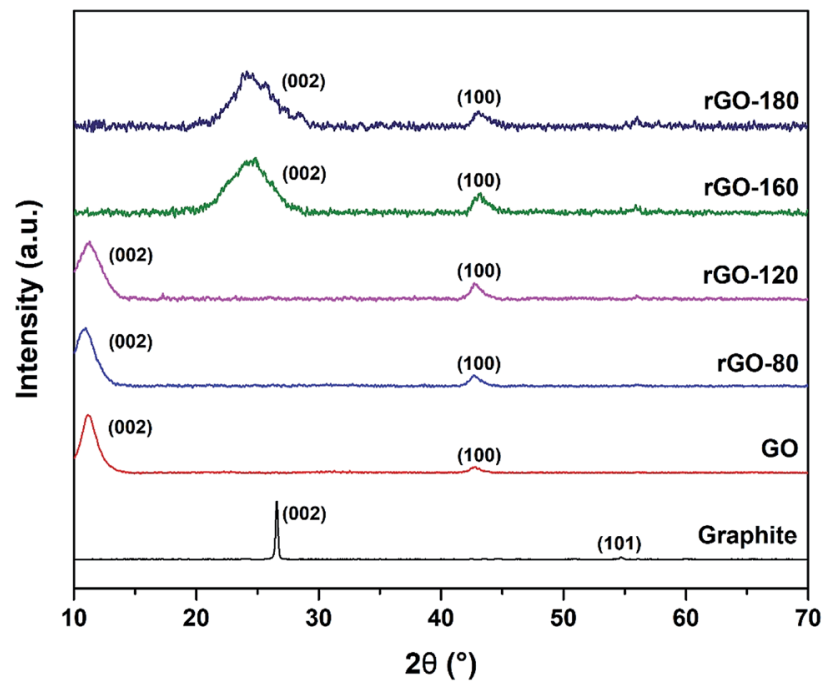

Fig. 3 XRD patterns of graphite, GO, rGO-80, rGO-120, rGO-160 and rGO-180.

(A)

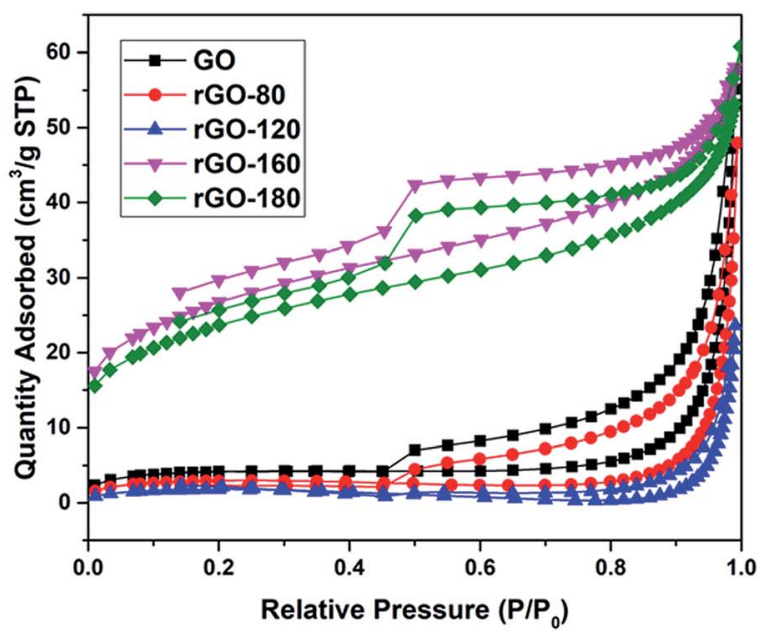

(B)

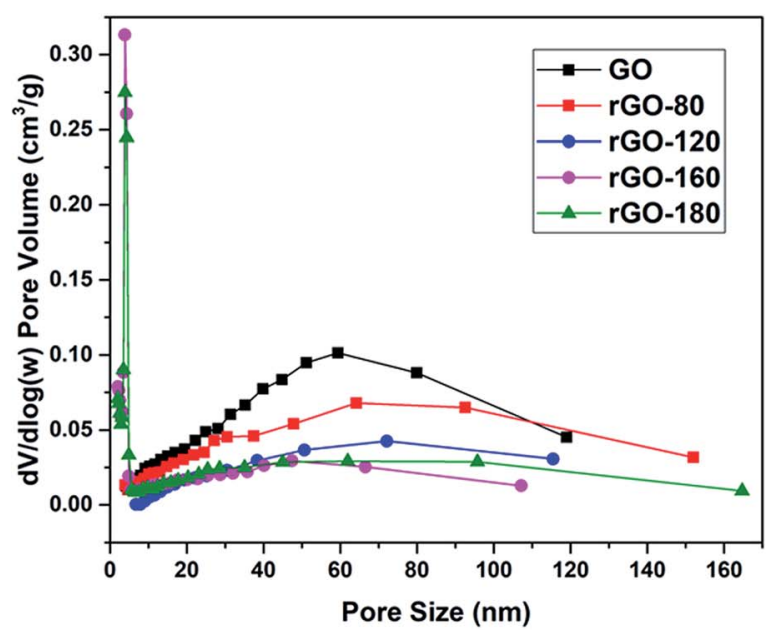

Fig. 4 (A) Nitrogen adsorption-desorption isotherms of GO, rGO-80, rGO-120, rGO-160 and rGO-180, (B) pore size distributions of GO, rGO-80, rGO-120, rGO-160 and rGO-180.
Table 2 BET/BJH textural parameters of GO, rGO-80, rGO-120, rGO160 and $\mathrm{rGO}-180$

\begin{tabular}{llll}
\hline Sample & $\begin{array}{l}\text { Surface area } \\
\left(\mathrm{m}^{2} \mathrm{~g}^{-1}\right)\end{array}$ & $\begin{array}{l}\text { Pore size } \\
(\mathrm{nm})\end{array}$ & $\begin{array}{l}\text { Pore volume } \\
\left(\mathrm{cm}^{3} \mathrm{~g}^{-1}\right)\end{array}$ \\
\hline GO & 15.03 & 59.42 & 0.085 \\
rGO-80 & 10.76 & 64.19 & 0.074 \\
rGO-120 & 6.67 & 72.18 & 0.037 \\
rGO-160 & 94.81 & 3.89 & 0.118 \\
rGO-180 & 83.81 & 3.90 & 0.094
\end{tabular}

found to increase, owing to the reduced oxygen content. ${ }^{36,37}$ At lower reduction temperatures of $80{ }^{\circ} \mathrm{C}$ and $120{ }^{\circ} \mathrm{C}$, the increment in $\mathrm{C} / \mathrm{O}$ atomic ratio was small. However, as the reduction temperature was elevated to $160^{\circ} \mathrm{C}$ and $180{ }^{\circ} \mathrm{C}$, the $\mathrm{C} / \mathrm{O}$ atomic

(A)

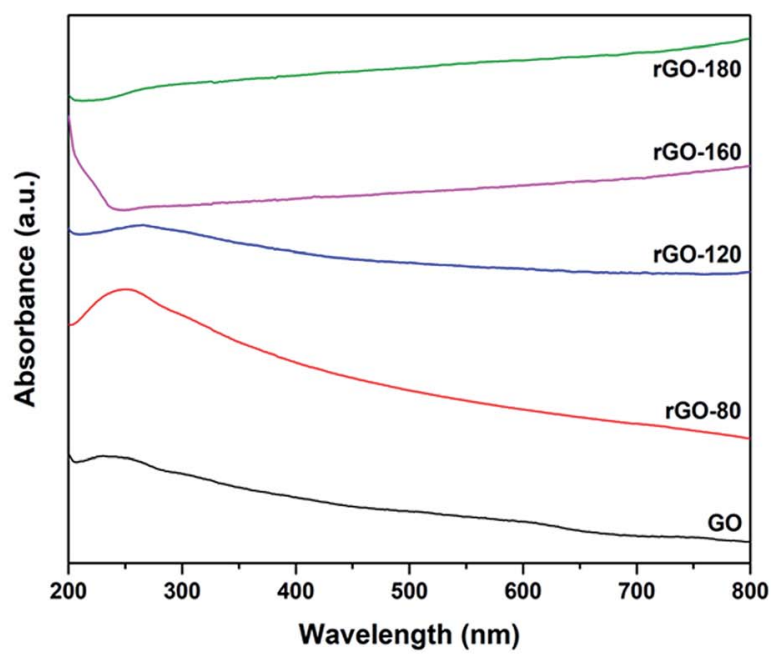

(B)

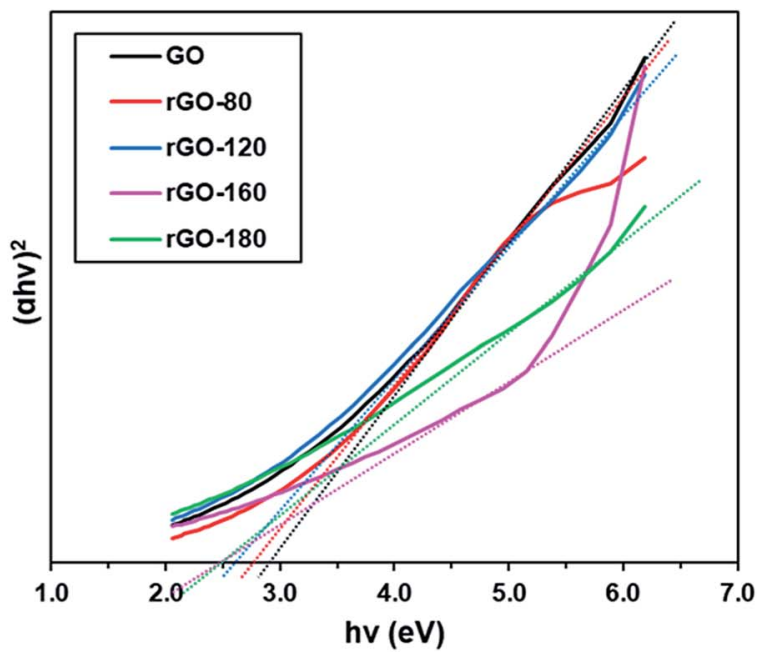

Fig. 5 (A) UV-vis absorption spectra of GO, rGO-80, rGO-120, rGO160 and rGO-180, (B) Tauc plots of GO, rGO-80, rGO-120, rGO-160 and rGO-180. 
(A)

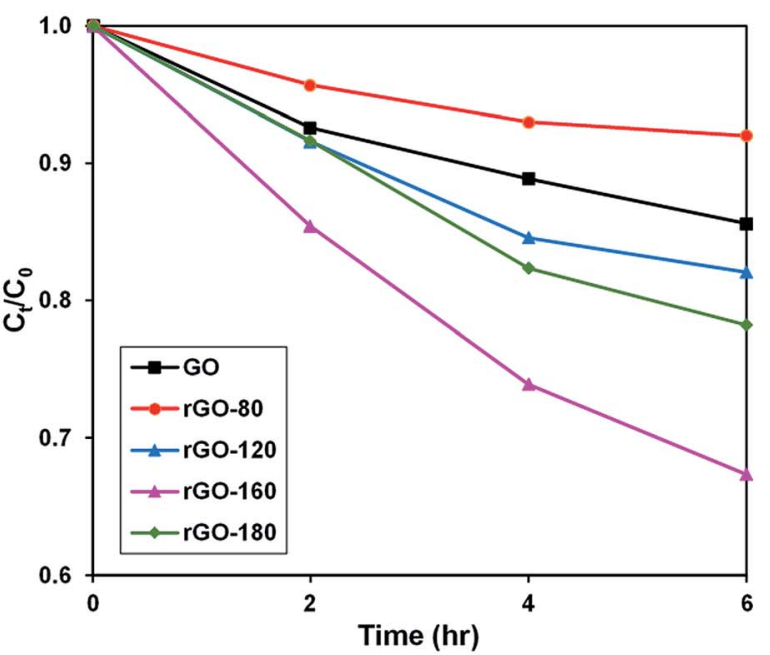

(B)

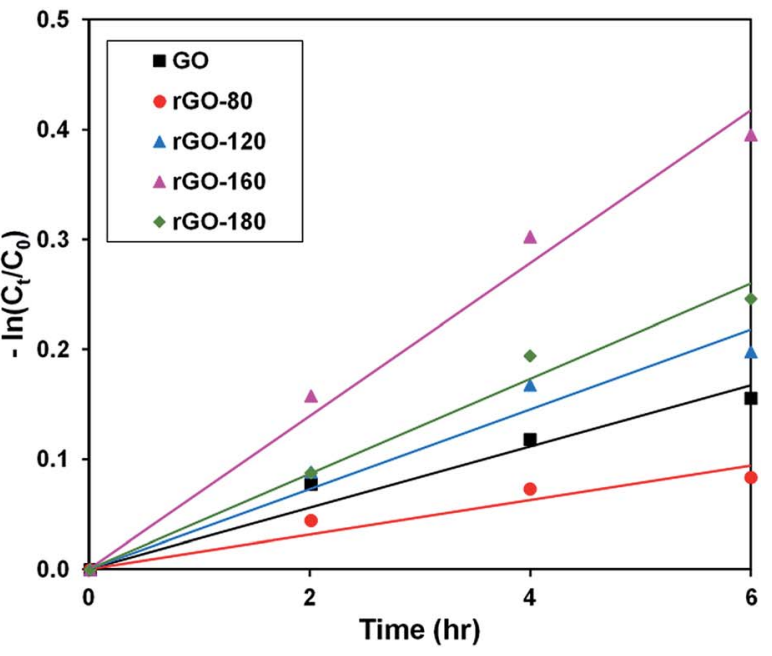

Fig. 6 (A) Photocatalytic degradation of MB dye normalized against adsorption, (B) pseudo-first order kinetic plot of $\mathrm{MB}$ dye, in the presence of GO, rGO-80, rGO-120, rGO-160 and rGO-180.

ratio was drastically increased. The EDX results concurred with the Raman data presented, whereby higher reduction temperature led to higher degree of GO reduction.

The XRD diffractograms of graphite, GO and rGOs are depicted in Fig. 3. An intense (002) peak and a very small (101) peak were observed in graphite at $2 \theta=26.57^{\circ}$ and $2 \theta=54.70^{\circ}$, respectively. Both peaks confirmed the structure of graphite and concurred with JCPDS card of $41-1487 .{ }^{38}$ Upon oxidation, the
(002) peak was shifted to $2 \theta=11.03^{\circ}$, which was attributed to the insertion of oxygenated functionalities and water molecules into the graphene layers. ${ }^{39}$ Nevertheless, the (002) peak of GO was still visible after $\mathrm{GO}$ was reduced at $80{ }^{\circ} \mathrm{C}$ and $120{ }^{\circ} \mathrm{C}$, possibly due to the low reduction temperatures, which mildly reduced GO to rGO. As the reduction temperature increased, the (002) peak of GO started to disappear while a new broader (002) peak of rGO began to emerge at $2 \theta=24.89^{\circ}$ for rGO-160 and $2 \theta$ $=23.85^{\circ}$ for rGO-180. The shifting of the (002) peak suggested the partial recovery of the $\mathrm{sp}^{2}$ carbon structure. ${ }^{40}$ Moreover, the (100) peak of GO at $2 \theta=42.63^{\circ}$ could also be detected in all rGOs, which reflected the turbostratic band of disordered carbon materials. ${ }^{12}$

The nitrogen physisorption isotherms of GO and rGOs are illustrated in Fig. 4A. All samples were found to show a type H3 hysteresis loop in a type II curve. ${ }^{41}$ A slope was present in each desorption curve, owing to the tensile strength effect that was produced by the instability of meniscus condensation inside the pores. $^{42}$ Both rGO-160 and rGO-180 had hysteresis loops that were partially closed and stopped at around $P / P_{0}=0.45$, possibly due to the presence of micropores in the samples. ${ }^{43}$ The pore size distribution curves of GO and rGOs are demonstrated in Fig. 4B, while the corresponding textural parameters are displayed in Table 2.

Based on Table 2, when GO was reduced from $80{ }^{\circ} \mathrm{C}$ to $120^{\circ} \mathrm{C}$, the total pore volume was reduced from 0.085 to 0.037 $\mathrm{cm}^{3} \mathrm{~g}^{-1}$, thus leading to a reduction of surface area. This was probably due to the aggregation effect of GO upon reduction. ${ }^{\mathbf{4 4 , 4 5}}$ A pronounced increment of surface area was then observed for rGO-160 at higher reduction temperature, owing to the significant removal of oxygen functional groups, ${ }^{46}$ causing the generation of higher pore volume. ${ }^{47}$ However, when the reduction temperature was increased to $180{ }^{\circ} \mathrm{C}$, the rGO sheets tended to stack together via $\pi-\pi$ interactions, resulting in the decreased surface area of rGO-180. ${ }^{48}$ Both rGO-160 and rGO-180 exhibited much smaller pore sizes and higher total pore volumes as compared to other samples.

Fig. 5A shows the UV-vis absorption spectra of GO and rGOs. At $230 \mathrm{~nm}$, a prominent absorption peak was observed in GO, which corresponded to the $\pi-\pi^{*}$ transition of aromatic $\mathrm{C}=\mathrm{C}$ bonds. ${ }^{49}$ When GO was reduced from $80{ }^{\circ} \mathrm{C}$ to $180{ }^{\circ} \mathrm{C}$, the peak location slowly moved to higher wavelengths. The red shifting of the peak indicated the partial restoration of the $\mathrm{sp}^{2}$ conjugation, thereby reducing the band gap. ${ }^{50}$ The optical band gaps of GO and rGOs were then determined from the linear fits of the Tauc plots, as

Table 3 Effect of reaction temperature on adsorption percentage, photocatalytic degradation efficiency, and photocatalytic degradation rate of MB dye

\begin{tabular}{llrr}
\hline Sample & Adsorption percentage (\%) & $\begin{array}{l}\text { Photocatalytic } \\
\text { degradation efficiency (\%) }\end{array}$ & $k\left(\mathrm{~h}^{-1}\right)$ \\
\hline GO & 10.07 & 14.41 & 0.028 \\
rGO-80 & 17.67 & 8.00 & 0.016 \\
rGO-120 & 11.60 & 17.96 & 0.036 \\
rGO-160 & 29.26 & 32.68 & 0.9075 \\
rGO-180 & 22.83 & 21.80 & 0.9516 \\
& & & 0.9840 \\
\end{tabular}


(A)

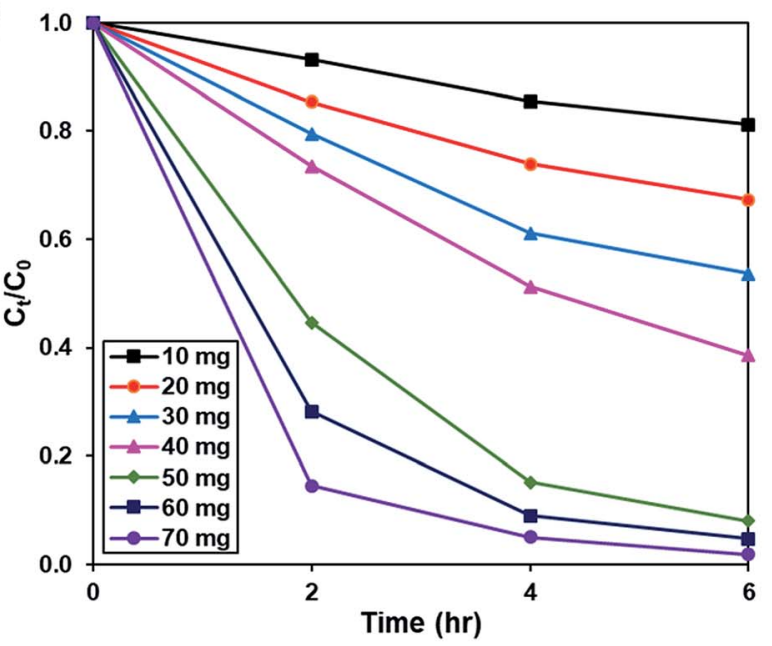

(B)

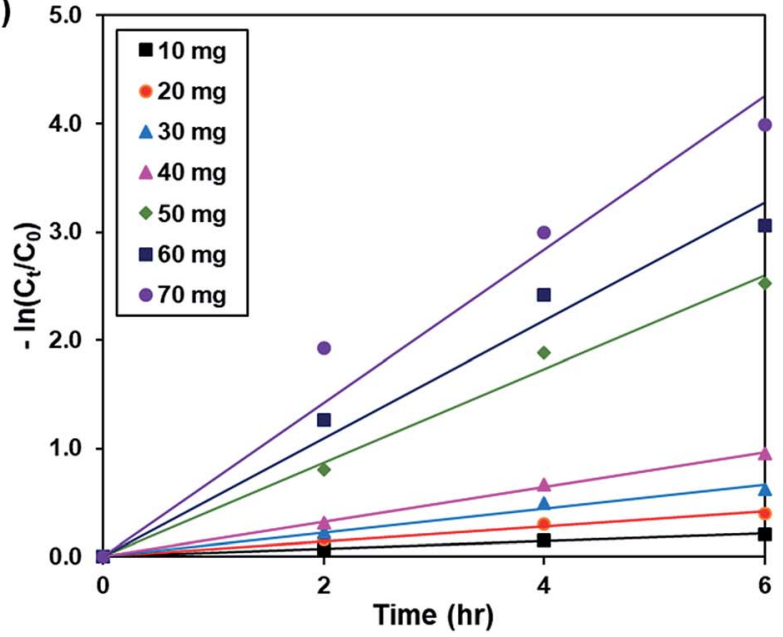

Fig. 7 (A) Effect of catalyst loading of $\mathrm{rGO}-160$ on photocatalytic degradation of $\mathrm{MB}$ dye normalized against adsorption, (B) pseudo-first order kinetic plot of $\mathrm{MB}$ dye, in the presence of $10 \mathrm{mg}, 20 \mathrm{mg}, 30 \mathrm{mg}$, $40 \mathrm{mg}, 50 \mathrm{mg}, 60 \mathrm{mg}$ and $70 \mathrm{mg}$ of rGO-160.

demonstrated in Fig. 5B. The band gap of GO was about $2.90 \mathrm{eV}$, concurring with the values reported in the existing literature. ${ }^{51,52}$

\subsection{Adsorption and photocatalytic activity measurements of GO and rGOs}

During the control experiment in the absence of photocatalyst, it was found that only $7.9 \%$ of MB dye was removed (Fig. S2†).
(A)

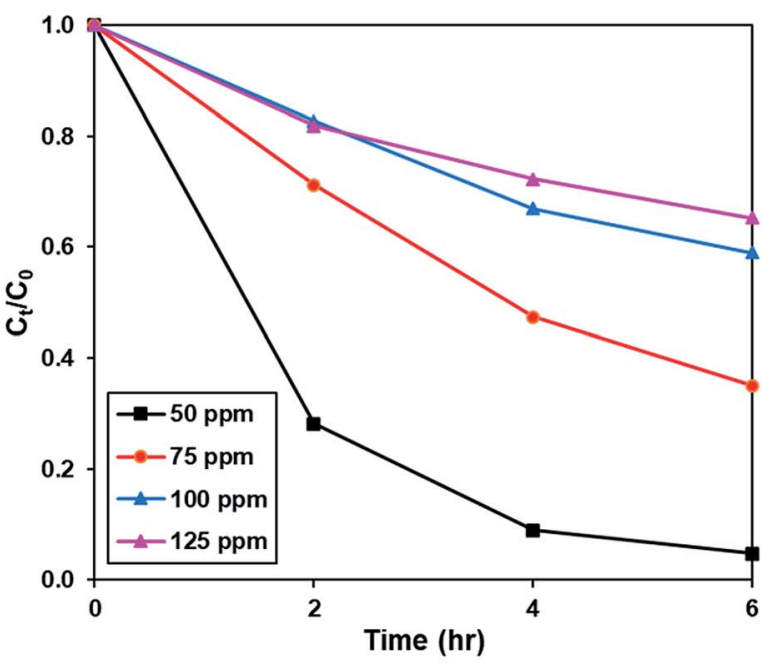

(B)

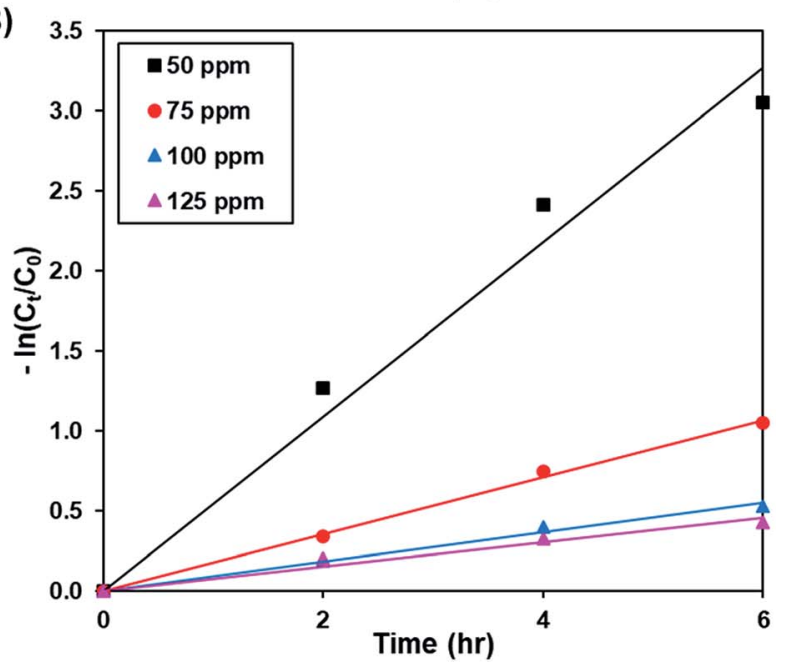

Fig. 8 (A) Effect of initial dye concentration of MB solution on photocatalytic degradation of $M B$ dye normalized against adsorption, (B) pseudo-first order kinetic plot of MB dye at 50 ppm, 75 ppm, 100 ppm and $125 \mathrm{ppm}$ of MB solution.

The slight removal of dye was due to photolysis effect, whereby similar results were also reported in previous studies. ${ }^{53,54}$

Upon stirring in the dark, the dye adsorption-desorption equilibrium on the photocatalysts was reached after 4 hours (Fig. S3-S6†). The photoactivity of GO and rGOs are illustrated in Fig. $6 \mathrm{~A}$ and $\mathrm{S} 7, \dagger$ while the pseudo-first-order fitting of photocatalytic dye degradation is depicted in Fig. 6B. The

Table 4 Effect of catalyst loading on adsorption percentage, photocatalytic degradation efficiency, and photocatalytic degradation rate of MB dye

\begin{tabular}{|c|c|c|c|c|}
\hline Weight (mg) & Adsorption percentage (\%) & $\begin{array}{l}\text { Photocatalytic } \\
\text { degradation efficiency (\%) }\end{array}$ & $k\left(\mathrm{~h}^{-1}\right)$ & $R^{2}$ \\
\hline 10 & 10.50 & 18.82 & 0.036 & 0.9919 \\
\hline 30 & 39.81 & 46.35 & 0.110 & 0.9822 \\
\hline 40 & 50.96 & 61.46 & 0.161 & 0.9983 \\
\hline 50 & 70.72 & 91.95 & 0.433 & 0.9914 \\
\hline
\end{tabular}


Table 5 Effect of initial dye concentration on adsorption percentage, photocatalytic degradation efficiency, and photocatalytic degradation rate of MB dye

\begin{tabular}{lllr}
\hline Concentration $(\mathrm{ppm})$ & Adsorption percentage (\%) & $\begin{array}{l}\text { Photocatalytic } \\
\text { degradation efficiency (\%) }\end{array}$ & $k\left(\mathrm{~h}^{-1}\right)$ \\
\hline 50 & 83.45 & 95.29 & 0.545 \\
75 & 52.25 & 64.97 & 0.978 \\
100 & 40.98 & 40.99 & 0.9972 \\
125 & 30.14 & 34.71 & 0.992 \\
& & & 0.076 \\
\end{tabular}

corresponding \% $C_{\text {ads }}, \% C_{\mathrm{deg}}, k$ and $R^{2}$ values are shown in Table 3. Since low reduction temperatures were insufficient for GO reduction, both rGO-80 and rGO-120 behaved more or less like GO in the dye adsorption process as compared to other rGOs. With a large surface area, rGO-160 was able to attain the highest adsorption of dye. When the reduction temperature
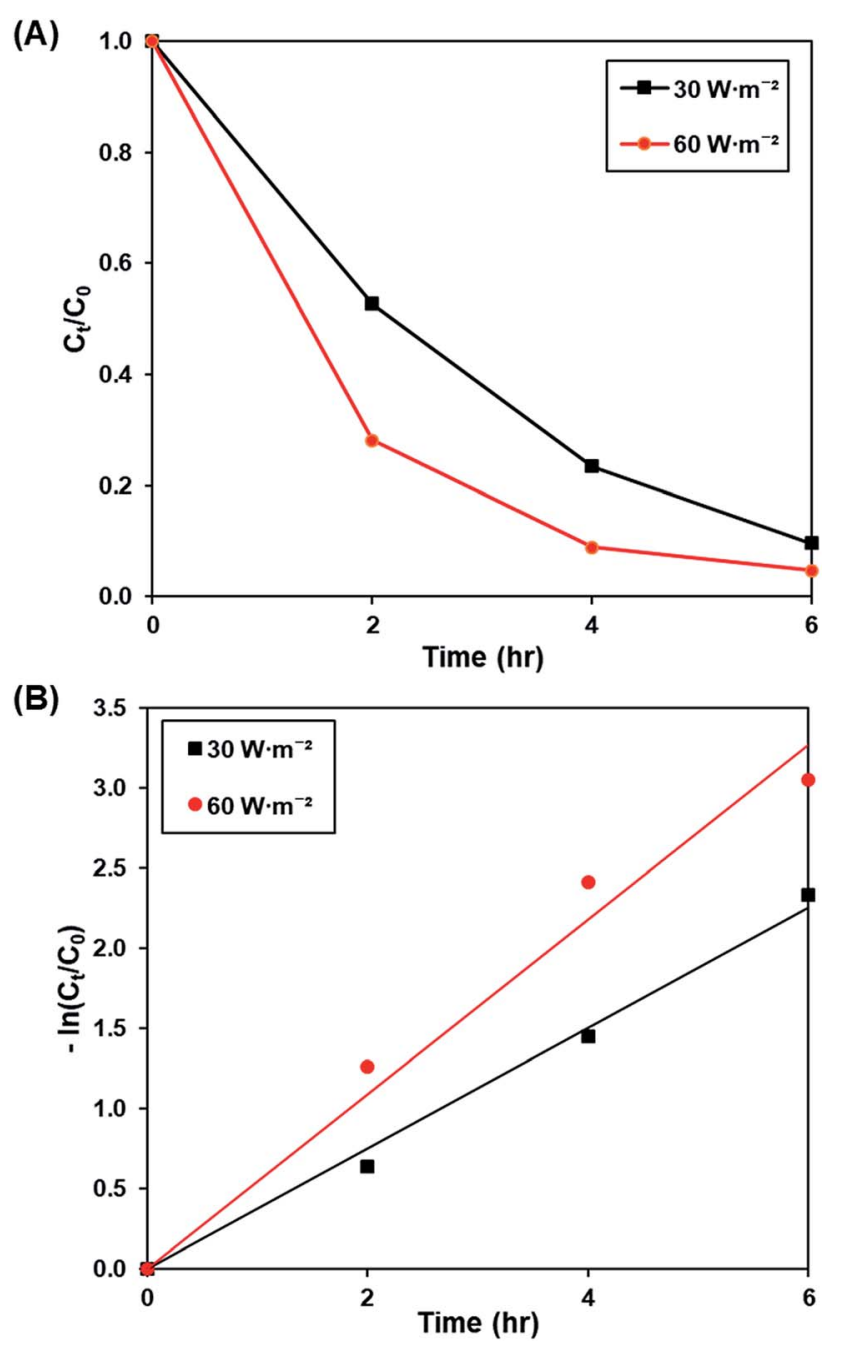

Fig. 9 (A) Effect of $30 \mathrm{~W} \mathrm{~m}^{-2}$ and $60 \mathrm{~W} \mathrm{~m}^{-2}$ of UV-C light source on photocatalytic degradation of $\mathrm{MB}$ dye normalized against adsorption, (B) pseudo-first order kinetic plot of $\mathrm{MB}$ dye at $30 \mathrm{~W} \mathrm{~m}^{-2}$ and $60 \mathrm{~W}$ $\mathrm{m}^{-2}$ of UV-C light source. increased to $180{ }^{\circ} \mathrm{C}$, there was a slight reduction in the amount of dye adsorbed owing to surface area reduction. As the irradiated light contained higher energy than the band gaps of GO and rGOs, electrons and holes were able to be generated, thereby providing ROS to break down the dye. Similar to GO, both rGO-80 and rGO-120 also demonstrated lower photoactivity as compared to other rGOs. The highest photoactivity was then exhibited by rGO-160. It was suggested that the dye molecules could interact better with the ROS when they were adsorbed on the surface as compared to moving freely in the aqueous solution. ${ }^{55}$ As rGO-180 exhibited smaller surface area than rGO-160, the available contact area for photocatalytic degradation reaction was also decreased.

\subsection{Optimization of adsorption and photocatalytic activity of rGO-160}

3.3.1. Effect of catalyst loading. Fig. $7 \mathrm{~A}$ and $\mathrm{S} 8 \dagger$ demonstrate the effect of catalyst dosage (10 to $70 \mathrm{mg}$ ) on the photoactivity of rGO-160, whereas the pseudo-first-order fitting of photocatalytic dye degradation is displayed in Fig. 7B. The corresponding \% $C_{\text {ads }}$ \% $C_{\text {deg }}, k$ and $R^{2}$ values are illustrated in Table 4 . The initial concentration of dye $(50 \mathrm{ppm})$, intensity of light $\left(60 \mathrm{~W} \mathrm{~m}^{-2}\right)$, and $\mathrm{pH}$ of solution $(\mathrm{pH} 6)$ were remained constant at all times. When the catalyst amount was increased, the total surface area of photocatalyst became larger. Subsequently, the available adsorption sites also increased, which in turn improved the dye adsorption process. ${ }^{56}$ Besides that, the total number of catalyst particles also increased, which then enhanced the absorption of photons for the production of electrons and holes, and improved the rate of formation of ROS for the decomposition of dye molecules. ${ }^{57}$ Despite the fact that the highest photoactivity was achieved by using $70 \mathrm{mg}$ of catalyst, the use of such high amount of catalyst almost provoked the complete removal of dye under dark condition. Therefore, $60 \mathrm{mg}$ was considered as the optimum catalyst loading for this work.

Table 6 Effect of light intensity on photocatalytic degradation efficiency and photocatalytic degradation rate of MB dye

\begin{tabular}{llll}
\hline $\begin{array}{l}\text { Light intensity } \\
\left(\mathrm{W} \mathrm{m}^{-2}\right)\end{array}$ & $\begin{array}{l}\text { Photocatalytic } \\
\text { degradation efficiency }(\%)\end{array}$ & $k\left(\mathrm{~h}^{-1}\right)$ & $R^{2}$ \\
\hline 30 & 90.31 & 0.377 & 0.9931 \\
60 & 95.29 & 0.545 & 0.9756
\end{tabular}


(A)

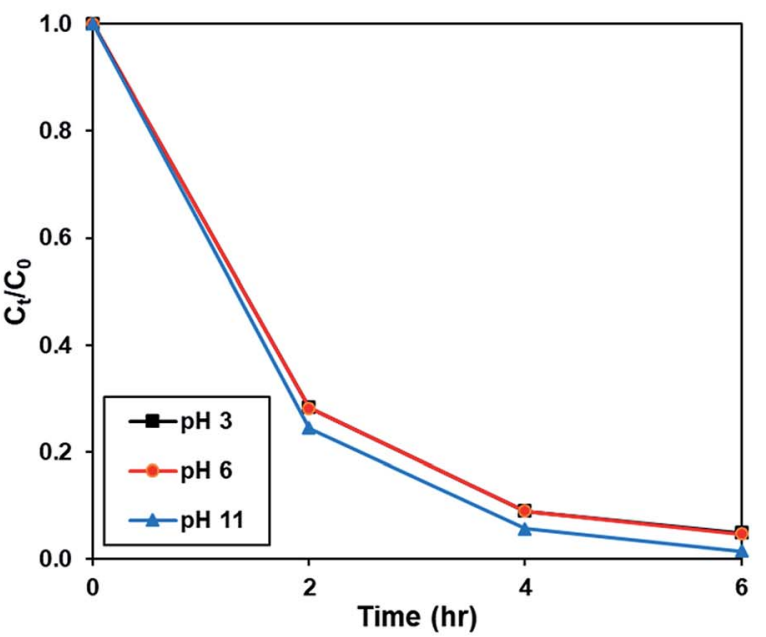

(B)

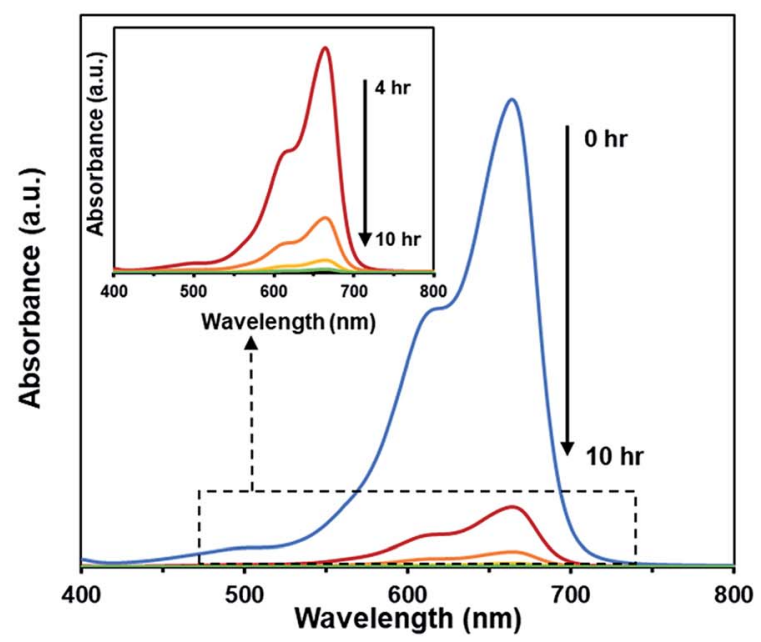

Fig. 10 (A) Effect of initial $\mathrm{pH}$ of dye solution at 3, 6, and 11 on photocatalytic degradation of MB dye normalized against adsorption, (B) time-dependent UV-vis absorption spectra for the adsorption and photocatalytic degradation of MB dye under optimized conditions.

3.3.2. Effect of initial dye concentration. Fig. $8 \mathrm{~A}$ and $\mathrm{S} 9 \dagger$ show the effect of initial concentration of dye (50 to $125 \mathrm{ppm}$ ) on the photoactivity of rGO-160, while the pseudo-first-order fitting of photocatalytic dye degradation is depicted in Fig. 8B. The corresponding \% $C_{\text {ads }}$, \% $C_{\text {deg }}, k$ and $R^{2}$ values are displayed in Table 5. Throughout the experiments, the catalyst loading (60 $\mathrm{mg}$ ), light intensity $\left(60 \mathrm{~W} \mathrm{~m}^{-2}\right)$, and $\mathrm{pH}$ of solution $(\mathrm{pH} 6)$ were kept constant. With the increase of initial concentration of dye solution, more dye molecules were accumulated on the catalyst surface. Eventually, the available adsorption sites became saturated and insufficient to accommodate the increasing amount of dye molecules. ${ }^{58}$ The production of ROS for the photocatalytic dye degradation reaction also became limited. ${ }^{\mathbf{9}}$ According to the Beer-Lambert law, the photocatalytic degradation rate decreased when the initial concentration of dye increased, owing to the shorter path length of photons entering the solution. ${ }^{60}$

3.3.3. Effect of light intensity. Fig. $9 \mathrm{~A}$ and $\mathrm{S} 10 \dagger$ illustrates the effect of light intensity $\left(30 \mathrm{~W} \mathrm{~m}^{-2}\right.$ and $\left.60 \mathrm{~W} \mathrm{~m}^{-2}\right)$ on the photoactivity of rGO-160, whereas the pseudo-first-order fitting of photocatalytic dye degradation is shown in Fig. 9B. The corresponding $\% C_{\mathrm{ads}}, \% C_{\mathrm{deg}}, k$ and $R^{2}$ values are demonstrated in Table 6 . It should be noted that the catalyst loading (60 mg), initial concentration of dye (50 ppm), and $\mathrm{pH}$ of solution ( $\mathrm{pH} \mathrm{6)}$ were kept constant at all times. When the light intensity was increased, there were more photons generated and absorbed by the photocatalyst. As a result, more electrons were readily excited to form more electrons and holes, which created more ROS for the breakdown of dye molecules. ${ }^{61}$

3.3.4. Effect of pH. Fig. $10 \mathrm{~A}$ and $\mathrm{S} 11 \dagger$ depict the effect of initial $\mathrm{pH}$ of solution ( $\mathrm{pH} \mathrm{3,6}$ and 11) on the photoactivity of rGO-160. The corresponding \% $C_{\text {ads }}$, \% $C_{\mathrm{deg}}, k$ and $R^{2}$ values are shown in Table 7. Throughout the experiments, the catalyst loading (60 mg), initial dye concentration (50 ppm), and light intensity $\left(60 \mathrm{~W} \mathrm{~m}^{-2}\right)$ were kept constant. Under acidic condition, there were more hydrogen ions present in the solution. The dye molecules then competed with the large amount of hydrogen ions for the unoccupied adsorption sites on the catalyst surface. ${ }^{62}$ It is known that MB dye can adsorb much better in basic medium as compared to acidic medium due to the cationic nature of the dye. ${ }^{63}$ In addition, the presence of excess hydroxyl ions also facilitated the formation of $\cdot \mathrm{OH}$, which in turn increased the photocatalytic degradation rate. ${ }^{64}$ The time-dependent UV-vis absorption spectra for the removal of dye under optimized conditions are illustrated in Fig. 10B.

3.3.5. Recyclability test. From the perspective of practical applications, the recyclability of a photocatalyst is considered a crucial factor. The stability of rGO-160 was investigated for the photocatalytic dye degradation reaction as a function of number of cycles, as shown in Fig. 11. After five consecutive runs, the $\% C_{\text {deg }}$ was still greater than $90 \%$, indicating that rGO-160 exhibited high stability and good reusability.

Table 7 Effect of $\mathrm{pH}$ on adsorption percentage, photocatalytic degradation efficiency, and photocatalytic degradation rate of MB dye

\begin{tabular}{lllr}
\hline $\mathrm{pH}$ & Adsorption percentage (\%) & $\begin{array}{l}\text { Photocatalytic } \\
\text { degradation efficiency (\%) }\end{array}$ & $k\left(\mathrm{~h}^{-1}\right)$ \\
3 & 81.85 & 95.14 & 0.541 \\
6 & 83.45 & 95.29 & 0.545 \\
11 & 87.39 & 98.57 & 0.9711
\end{tabular}




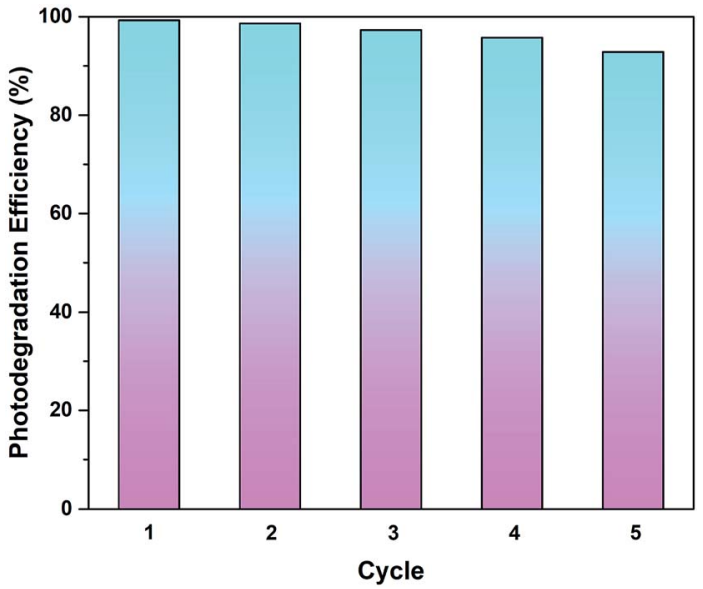

Fig. 11 Recycling tests of rGO-160 for the photocatalytic degradation of MB dye.

\section{Conclusion}

GO was successfully reduced in the absence of toxic reductants via a facile one-pot solvothermal approach. At $160{ }^{\circ} \mathrm{C}$, the $\mathrm{sp}^{2}$ hybridized structure of graphene was partially restored, following the removal of surficial oxygen functionalities. The best adsorptive removal $(29.26 \%)$ and photocatalytic degradation $(32.68 \%)$ of MB dye were then achieved by rGO-160, which revealed that the enlargement of surface area and the narrowing of band gap were the main reasons. By optimizing the amount of catalyst to $60 \mathrm{mg}$, initial dye concentration to $50 \mathrm{ppm}$, light intensity to $60 \mathrm{~W} \mathrm{~m}^{-2}$, and solution $\mathrm{pH}$ to 11 , the adsorption (87.39\%) and photocatalytic activity (98.57\%) of rGO-160 were further enhanced. After repeating the reaction for up to five times, rGO-160 was still able to degrade more than $90 \%$ of $\mathrm{MB}$ dye, indicating that it was highly stable and reusable.

\section{Conflicts of interest}

There are no conflicts to declare.

\section{Acknowledgements}

This research work was financially supported by the University Malaya Research Grant (no. RP045B-17AET), and ImpactOriented Interdisciplinary Research Grant (no. IIRG018A-2019).

\section{References}

1 A. Mohamed, R. El-Sayed, T. Osman, M. Toprak, M. Muhammed and A. Uheida, Environ. Res., 2016, 145, 18-25.

2 S. Li, Q. Lin, X. Liu, L. Yang, J. Ding, F. Dong, Y. Li, M. Irfan and P. Zhang, RSC Adv., 2018, 8, 20277-20286.

3 M. Thakur, G. Sharma, T. Ahamad, A. A. Ghfar, D. Pathania and M. Naushad, Colloids Surf., B, 2017, 157, 456-463.

4 G. Z. Kyzas and M. Kostoglou, Materials, 2014, 7, 333-364.
5 Y. Huang, S. S. H. Ho, Y. Lu, R. Niu, L. Xu, J. Cao and S. Lee, Molecules, 2016, 21, 56.

6 K. M. Lee, C. W. Lai, K. S. Ngai and J. C. Juan, Water Res., 2016, 88, 428-448.

7 A. Sandoval, C. Hernández-Ventura and T. E. Klimova, Fuel, 2017, 198, 22-30.

8 C. Marichy, P. A. Russo, M. Latino, J.-P. Tessonnier, M.-G. Willinger, N. Donato, G. Neri and N. Pinna, J. Phys. Chem. C, 2013, 117, 19729-19739.

9 C. Hu, T. Lu, F. Chen and R. Zhang, J. Chin. Adv. Mater. Soc., 2013, 1, 21-39.

10 S. J. Rowley-Neale, E. P. Randviir, A. S. A. Dena and C. E. Banks, Appl. Mater. Today, 2017, 10, 218-226.

11 Q. A. Khan, A. Shaur, T. A. Khan, Y. F. Joya and M. Awan, Cogent Chem., 2017, 3, 1298980.

12 T. F. Emiru and D. W. Ayele, Egypt. J. Basic Appl. Sci., 2017, 4, 74-79.

13 S. Thangavel and G. Venugopal, Powder Technol., 2014, 257, 141-148.

14 M. Zhang, Q. Yao, C. Lu, Z. Li and W. Wang, ACS Appl. Mater. Interfaces, 2014, 6, 20225-20233.

15 A. J. Kumar and C. Namasivayam, Sustainable Environ. Res., 2014, 24, 73-80.

16 K. Gupta and O. P. Khatri, J. Colloid Interface Sci., 2017, 501, 11-21.

17 N. M. Mahmoodi, S. M. Maroofi, M. Mazarji and G. NabiBidhendi, J. Surfactants Deterg., 2017, 20, 1085-1093.

18 S. Cui, H. Pu, E. C. Mattson, Z. Wen, J. Chang, Y. Hou, C. J. Hirschmugl and J. Chen, Anal. Chem., 2014, 86, 75167522.

19 C. Minitha, M. Lalitha, Y. Jeyachandran and L. Senthilkumar, Mater. Chem. Phys., 2017, 194, 243-252.

$20 \mathrm{H}$. Zhu, Y. Fu, R. Jiang, J. Yao, L. Liu, Y. Chen, L. Xiao and G. Zeng, Appl. Surf. Sci., 2013, 285, 865-873.

21 T. Liu, L. Wang, X. Lu, J. Fan, X. Cai, B. Gao, R. Miao, J. Wang and Y. Lv, RSC Adv., 2017, 7, 12292-12300.

22 C. Zhao, Q. Wang, H. Zhang, S. Passerini and X. Qian, ACS Appl. Mater. Interfaces, 2016, 8, 15661-15667.

23 J. Cheng, R. Zhong, J. Lin, J. Zhu, W. Wan and X. Chen, Materials, 2017, 10, 443.

24 V. Georgakilas, J. A. Perman, J. Tucek and R. Zboril, Chem. Rev., 2015, 115, 4744-4822.

25 A. Morais, C. Longo, J. R. Araujo, M. Barroso, J. R. Durrant and A. F. Nogueira, Phys. Chem. Chem. Phys., 2016, 18, 2608-2616.

26 C. Liu, X. Wang, P. Ma, J. Chen, J. Jiang, Y. Ai, E. Wang, S. Han and S. Rong, RSC Adv., 2015, 5, 57383-57388.

27 G. Ali, A. Mehmood, H. Y. Ha, J. Kim and K. Y. Chung, Sci. Rep., 2017, 7, 40910.

28 K. Spilarewicz-Stanek, A. Kisielewska, J. Ginter, K. Bałuszyńska and I. Piwoński, RSC Adv., 2016, 6, 6005660067.

29 S. M. Tan, A. Ambrosi, C. K. Chua and M. Pumera, J. Mater. Chem. A, 2014, 2, 10668-10675.

30 X. Mei, X. Meng and F. Wu, Phys. E, 2015, 68, 81-86.

31 X. H. Tai, S. W. Chook, C. W. Lai, K. M. Lee, T. C. K. Yang, S. Chong and J. C. Juan, RSC Adv., 2019, 9, 18076-18086. 
32 C. Nethravathi and M. Rajamathi, Carbon, 2008, 46, 19941998.

33 Q. Zhuo, J. Gao, M. Peng, L. Bai, J. Deng, Y. Xia, Y. Ma, J. Zhong and X. Sun, Carbon, 2013, 52, 559-564.

34 Z. Li, S. C. Xu, C. Zhang, X. Y. Liu, S. S. Gao, L. T. Hu, J. Guo, Y. Ma, S. Z. Jiang and H. P. Si, Sci. Rep., 2016, 6, 38539.

35 P. Cui, J. Lee, E. Hwang and H. Lee, Chem. Commun., 2011, 47, 12370-12372.

36 A. AlShammari, M. M. Halim, F. K. Yam, N. H. Al-Hardan, N. H. M. Kaus, K. Umar, T. F. Qahtan and M. N. M. Ibrahim, Phys. B, 2019, 572, 296-301.

37 P. Phukan, R. Narzary and P. P. Sahu, Mater. Sci. Semicond. Process., 2019, 104, 104670.

38 M. Fathy, A. Gomaa, F. A. Taher, M. M. El-Fass and A. E.-H. B. Kashyout, J. Mater. Sci., 2016, 51, 5664-5675.

39 V. Loryuenyong, K. Totepvimarn, P. Eimburanapravat, W. Boonchompoo and A. Buasri, Adv. Mater. Sci. Eng., 2013, 2013, 923403.

40 G. Pavoski, T. Maraschin, F. d. C. Fim, N. M. Balzaretti, G. B. Galland, C. S. Moura and N. R. d. S. Basso, Mater. Res., 2017, 20, 53-61.

41 M. Thommes, K. Kaneko, A. V. Neimark, J. P. Olivier, F. Rodriguez-Reinoso, J. Rouquerol and K. S. Sing, Pure Appl. Chem., 2015, 87, 1051-1069.

42 Z. A. ALOthman, Materials, 2012, 5, 2874-2902.

43 H. Moussa, E. Girot, K. Mozet, H. Alem, G. Medjahdi and R. Schneider, Appl. Catal., B, 2016, 185, 11-21.

44 H. Su, C. Zhang, X. Li, L. Wu and Y. Chen, RSC Adv., 2018, 8, 39140-39148.

45 I. Chowdhury, N. D. Mansukhani, L. M. Guiney, M. C. Hersam and D. Bouchard, Environ. Sci. Technol., 2015, 49, 10886-10893.

46 B. Zhao, P. Liu, Y. Jiang, D. Pan, H. Tao, J. Song, T. Fang and W. Xu, J. Power Sources, 2012, 198, 423-427.

47 G. Srinivas, J. Burress and T. Yildirim, Energy Environ. Sci., 2012, 5, 6453-6459.
48 N. Morimoto, T. Kubo and Y. Nishina, Sci. Rep., 2016, 6, 21715.

49 S. Xu, L. Yong and P. Wu, ACS Appl. Mater. Interfaces, 2013, 5, 654-662.

50 S. Saxena, T. A. Tyson, S. Shukla, E. Negusse, H. Chen and J. Bai, Appl. Phys. Lett., 2011, 99, 013104.

51 P. Kumar, B. Sain and S. L. Jain, J. Mater. Chem. A, 2014, 2, 11246-11253.

52 Q. Zhang, H. Zheng, Z. Geng, S. Jiang, J. Ge, K. Fan, S. Duan, Y. Chen, X. Wang and Y. Luo, J. Am. Chem. Soc., 2013, 135, 12468-12474.

53 A. A. Mansur, H. S. Mansur, F. P. Ramanery, L. C. Oliveira and P. P. Souza, Appl. Catal., B, 2014, 158, 269-279.

54 M. B. Akin and M. Oner, Ceram. Int., 2013, 39, 9759-9762.

55 S. He, P. Hou, E. Petropoulos, Y. Feng, Y. Yu, L. Xue and L. Yang, Front. Chem., 2018, 6, 219.

56 H. Naeem, M. Ajmal, S. Muntha, J. Ambreen and M. Siddiq, RSC Adv., 2018, 8, 3599-3610.

57 D. Rajamanickam and M. Shanthi, Arabian J. Chem., 2016, 9, S1858-S1868.

58 R. Sahraei, K. Hemmati and M. Ghaemy, RSC Adv., 2016, 6, 72487-72499.

59 Y. Gao, S. Gu, L. Duan, Y.-r. Wang and G. Gao, Soft Matter, 2019, 15, 3897-3905.

60 C.-S. Lu, C.-C. Chen, L.-K. Huang, P.-A. Tsai and H.-F. Lai, Catalysts, 2013, 3, 501-516.

61 W. Anku, S. O.-B. Oppong, S. K. Shukla and P. P. Govender, Acta Chim. Slov., 2016, 63, 380-391.

62 T. Huang, M. Yan, K. He, Z. Huang, G. Zeng, A. Chen, M. Peng, H. Li, L. Yuan and G. Chen, J. Colloid Interface Sci., 2019, 543, 43-51.

63 M. Zarrabi, M. Haghighi, R. Alizadeh and S. Mahboob, Sep. Purif. Technol., 2019, 211, 738-752.

64 Y.-J. Chiang and C.-C. Lin, Powder Technol., 2013, 246, 137143. 